\title{
Population Pharmacokinetic/Pharmacodynamic Analysis of Alirocumab in Healthy Volunteers or Hypercholesterolemic Subjects Using an Indirect Response Model to Predict Low- Density Lipoprotein Cholesterol Lowering: Support for a Biologics License Application Submission: Part II
}

\author{
Xavier Nicolas $^{1} \cdot$ Nassim Djebli $^{1} \cdot$ Clémence Rauch $^{1} \cdot$ Aurélie Brunet $^{1} \cdot$ \\ Fabrice Hurbin ${ }^{1} \cdot J^{\prime}$ ean-Marie Martinez ${ }^{1} \cdot$ David Fabre $^{1}$ \\ Published online: 3 May 2018 \\ (C) The Author(s) 2018
}

\begin{abstract}
Background Alirocumab, a human monoclonal antibody against proprotein convertase subtilisin/kexin type 9 (PCSK9), significantly lowers low-density lipoprotein cholesterol levels.

Objective This analysis aimed to develop and qualify a population pharmacokinetic/pharmacodynamic model for alirocumab based on pooled data obtained from 13 phase $\mathrm{I} / \mathrm{II} / \mathrm{III}$ clinical trials.

Methods From a dataset of 2799 individuals (14,346 lowdensity lipoprotein-cholesterol values), individual pharmacokinetic parameters from the population pharmacokinetic model presented in Part I of this series were used to estimate alirocumab concentrations. As a second step, we then developed the current population pharmacokinetic/ pharmacodynamic model using an indirect response model with a Hill coefficient, parameterized with increasing lowdensity lipoprotein cholesterol elimination, to relate alirocumab concentrations to low-density lipoprotein cholesterol values.

Results The population pharmacokinetic/pharmacodynamic model allowed the characterization of the pharmacokinetic/pharmacodynamic properties of alirocumab in the target population and estimation of individual low-
\end{abstract}

density lipoprotein cholesterol levels and derived pharmacodynamic parameters (the maximum decrease in lowdensity lipoprotein cholesterol values from baseline and the difference between baseline low-density lipoprotein cholesterol and the pre-dose value before the next alirocumab dose). Significant parameter-covariate relationships were retained in the model, with a total of ten covariates (sex, age, weight, free baseline PCSK9, total time-varying PCSK9, concomitant statin administration, total baseline PCSK9, co-administration of high-dose statins, disease status) included in the final population pharmacokinetic/pharmacodynamic model to explain betweensubject variability. Nevertheless, the high number of covariates included in the model did not have a clinically meaningful impact on model-derived pharmacodynamic parameters.

Conclusions This model successfully allowed the characterization of the population pharmacokinetic/pharmacodynamic properties of alirocumab in its target population and the estimation of individual low-density lipoprotein cholesterol levels.
Electronic supplementary material The online version of this article (https://doi.org/10.1007/s40262-018-0670-5) contains supplementary material, which is available to authorized users.

Xavier Nicolas

xavier.nicolas@sanofi.com

1 TMED/PKDM Department, Sanofi, 371 Rue du Professeur Joseph Blayac, 34184 Montpellier Cedex 04, France 


\section{Key Points}

The population pharmacokinetic/pharmacodynamic model of alirocumab successfully described the relationship between alirocumab concentrations and low-density lipoprotein cholesterol (LDL-C) levels in the target population of 2799 healthy volunteers or patients from 13 phase I/II/III clinical studies.

The population pharmacokinetic/pharmacodynamic model allowed the estimation of individual LDL-C levels and derived pharmacodynamic parameters (the maximum decrease in LDL-C values from baseline and the difference between baseline LDL-C and the pre-dose value before the next alirocumab dose).

Ten covariates were included in the final model, all exhibiting moderate to strong effects on the model parameters; however, although some covariates were associated with more frequent dose increases to accommodate for the difference in response, the resulting LDL-C reduction was similar regardless of the covariate.

\section{Introduction}

Atherosclerosis is a disease in which plaques develop on the inner arterial wall, leading to progressive hardening and narrowing of the arteries and resulting in a multitude of diseases, such as coronary heart disease, cerebrovascular disease, and peripheral vascular disease. Atherogenesis is initiated and promoted by low-density lipoprotein cholesterol (LDL-C); a high level of circulating LDL-C is strongly associated with the risk of developing atherosclerotic lesions and adverse cardiovascular outcomes [1]. Lowering LDL-C has been associated with a reduction in the risk of cardiovascular disease [2], resulting in statistically significant and clinically important reductions in the rates of cardiovascular events for patients $[1,3]$.

Currently, statins are the first-line therapy to reduce LDL-C for patients at high risk of cardiovascular disease $[4,5]$. Although they have a generally good safety profile, statin use is associated with adverse symptoms in some individuals, the most common being muscle-related side effects, with myopathy reported in severe cases [6-8]. For patients with insufficient LDL-C reduction with statin therapy, recent updates to lipid management guidelines in
Europe and the USA have proposed that additional nonstatin therapies such as ezetimibe (a cholesterol absorption inhibitor) or an inhibitor to proprotein convertase subtilisin/kexin type 9 (PCSK9) may be justified, depending on the patient's risk of cardiovascular disease [3, 4].

Proprotein convertase subtilisin/kexin type 9 is a member of the subtilisin family of serine proteases expressed primarily in the liver $[9,10]$. Recent studies indicate that PCSK9 binds directly to the low-density lipoprotein receptor (LDL-R) and promotes LDL-R internalization and degradation [11]. This results in a lower number of LDL-Rs on the surface of hepatocytes and a decrease in the ability of the liver to remove low-density lipoprotein particles from the circulation, leading to greater serum LDL-C levels [12-14]. Alirocumab, a fully human monoclonal antibody to PCSK9, binds to and inhibits the PCSK9-mediated internalization of LDL-Rs [15].

Alirocumab has recently been approved by the US Food and Drug Administration as an adjunct to diet and maximally tolerated statin therapy in adults with clinical atherosclerotic cardiovascular disease or heterozygous familial hypercholesterolemia who require additional LDL$\mathrm{C}$ lowering. The European Medicines Agency has approved alirocumab for those with non-familial and heterozygous familial primary hypercholesterolemia or mixed dyslipidemia, as an adjunct to diet in combination with statins \pm other lipid-lowering therapies in patients unable to reach LDL-C goals with the maximally tolerated dose of a statin, who are statin intolerant, or for whom a statin is contraindicated [16, 17]. Significant reductions in LDL-C of up to $61 \%$ have been reported in patients with hypercholesterolemia when treated with alirocumab used in combination with statins [18-24]. As with other monoclonal antibodies, alirocumab has a non-linear pharmacokinetic profile; this is characterized by a target-mediated drug disposition (TMDD) process $[25,26]$ whereby the kinetics are a function of its mechanism of action.

This current analysis aimed to develop and validate a population pharmacokinetic/pharmacodynamic (PopPK/ PD) model able to predict individual LDL-C levels as a function of the individual pharmacokinetic parameters of alirocumab. We evaluated the relationship between alirocumab concentrations, estimated from the PopPK model presented in Part I of this paper [27], and LDL-C levels in healthy individuals and patients with familial and non-familial hypercholesterolemia. Potential relationships between population pharmacodynamic parameters and demographic covariates, free and total PCSK9 levels, relevant co-administered therapies, disease status, and relevant biologic constants were also evaluated. 


\section{Methods}

\subsection{Clinical Study Design}

The dataset comprised data from 13 clinical studies conducted in healthy volunteers with elevated LDL-C levels and patients with familial or non-familial hypercholesterolemia $(n=2799)$, including five phase I studies (NCT01026597, NCT01074372, NCT01161082 [28], NCT01448317 [29], and NCT01723735 [30]), four phase II studies (NCT01288469 [31], NCT01288443 [32], NCT01812707 [29], and NCT01266876 [33]), and four phase III studies (COMBO II [NCT01644188] [21], MONO [NCT01644474] [34], FH I [NCT01623115] [18], and LONG TERM [NCT01507831] [19]).

Alirocumab was administered either as a single subcutaneous dose, or as multiple subcutaneous doses, except for one study with a single intravenous dose. The dose of alirocumab ranged from $0.3 \mathrm{mg} / \mathrm{kg}$ up to $12 \mathrm{mg} / \mathrm{kg}$ for the intravenous study, and from $50 \mathrm{mg}$ up to $300 \mathrm{mg}$ for the subcutaneous studies; doses were administered every 2 weeks $(\mathrm{Q} 2 \mathrm{~W})$ or every 4 weeks over a treatment period of up to 2 years. A summary of the clinical trials included in the analysis is provided in Table 1.

\subsection{Analytical Methods}

Serum LDL-C levels were calculated using the Friedewald formula for all studies [35]. The concentration of total alirocumab in human serum was determined using an enzyme-linked immunosorbent assay, as described previously [30]. Further details regarding the validated enzymelinked immunosorbent assay and analysis of lipid samples are provided in the Supplementary Methods and Table 1 of the Electronic Supplementary Material (ESM).

\subsection{Pharmacokinetic Exclusion Criteria}

Patients and healthy volunteers who received placebo in the clinical trials were excluded from the overall database of randomized and treated subjects. Of the remaining participants who received alirocumab, data were excluded because of missing LDL-C levels, missing alirocumab concentrations, or values below the lower limit of quantification. Missing covariate values were replaced by the previous value from the same individual (last observation carried forward). The baseline values of free and total PCSK9 levels were calculated as the mean of all measurements taken before administration of the first dose (including those taken during screening).

\subsection{Population Pharmacokinetic/Pharmacodynamic Model Development}

The PopPK/PD model was developed using the NONMEM computer program (version 7.2; ICON Development Solutions, San Antonio, TX, USA) running on a LINUX cluster of multi-processor computers [36]. All runs were performed using the first-order conditional estimation method with interaction option. A two-step procedure was used to develop the PopPK/PD model. First, individual pharmacokinetic parameters from the previously developed population pharmacokinetic model (a Michaelis-Menten approximation of a TMDD model) described in Part I [27] were used to estimate total alirocumab concentrations at all time points needed for the development of the PopPK/PD model. Second, the PopPK/PD relationship was analyzed using a turnover model for LDL-C levels; an indirect response model (type IV, stimulation of loss of response) with a Hill coefficient was used to link total alirocumab concentrations with LDL-C [37]. This first model constituted the pharmacostatistical model. Although the effects of alirocumab on LDL-C are mediated through inhibition of PCSK9, the PCSK9 level was not used to develop the model. Instead, it was assessed as a covariate. Low-density lipoprotein cholesterol was chosen to develop the PopPK/ PD model as it is a parameter with demonstrated clinical impact, which is routinely measured in clinical practice.

\subsection{Covariate Screening and Inclusion Strategy}

In the pharmacostatistical model, population pharmacodynamic parameters (fixed and random effects) were first computed assuming no dependency between pharmacodynamic parameters and covariates. Following this, the impact of various covariates ( 16 in total) on the individual pharmacodynamic estimates was investigated. Demographic characteristics including body weight, body mass index, age, sex, disease status (heterozygous familial/nonfamilial hypercholesterolemia subjects vs. healthy subjects), albumin levels, and baseline LDL-C levels were tested as possible model covariates. Additional covariates tested included co-administration of relevant background therapies (statins, fibrates, or ezetimibe), free and total serum PCSK9 levels (both at baseline and time varying), and statin dose. When possible, covariate information available during the course of the trial (time-varying values) were used. Descriptive statistics of the baseline characteristics of all individuals included in the data set are presented in Table 2 of the ESM.

The selected covariates were added individually to the model (forward selection method) and tested for statistical significance on the objective function value (OFV). Covariates that produced a significant change $(p<0.05$ 


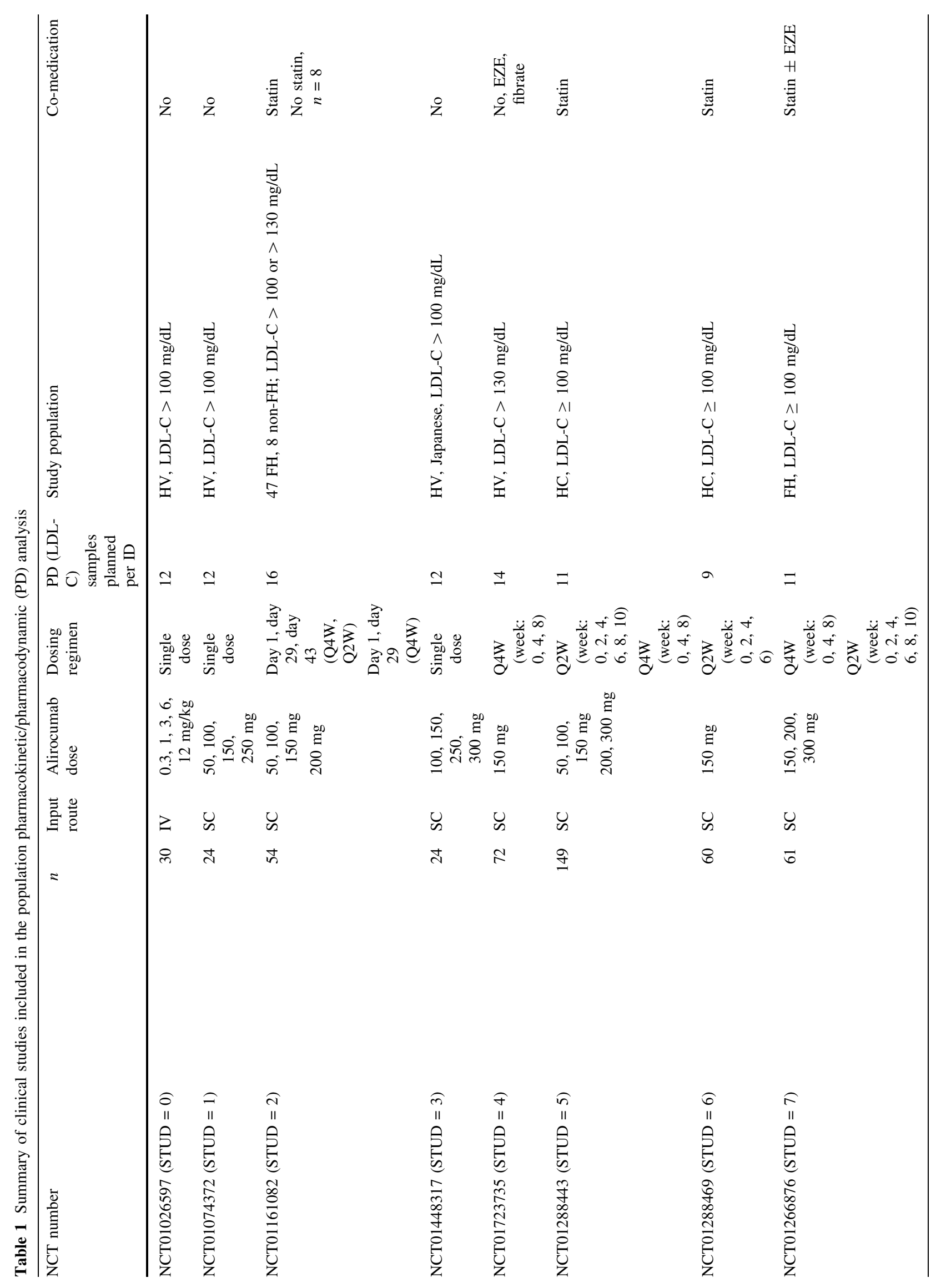




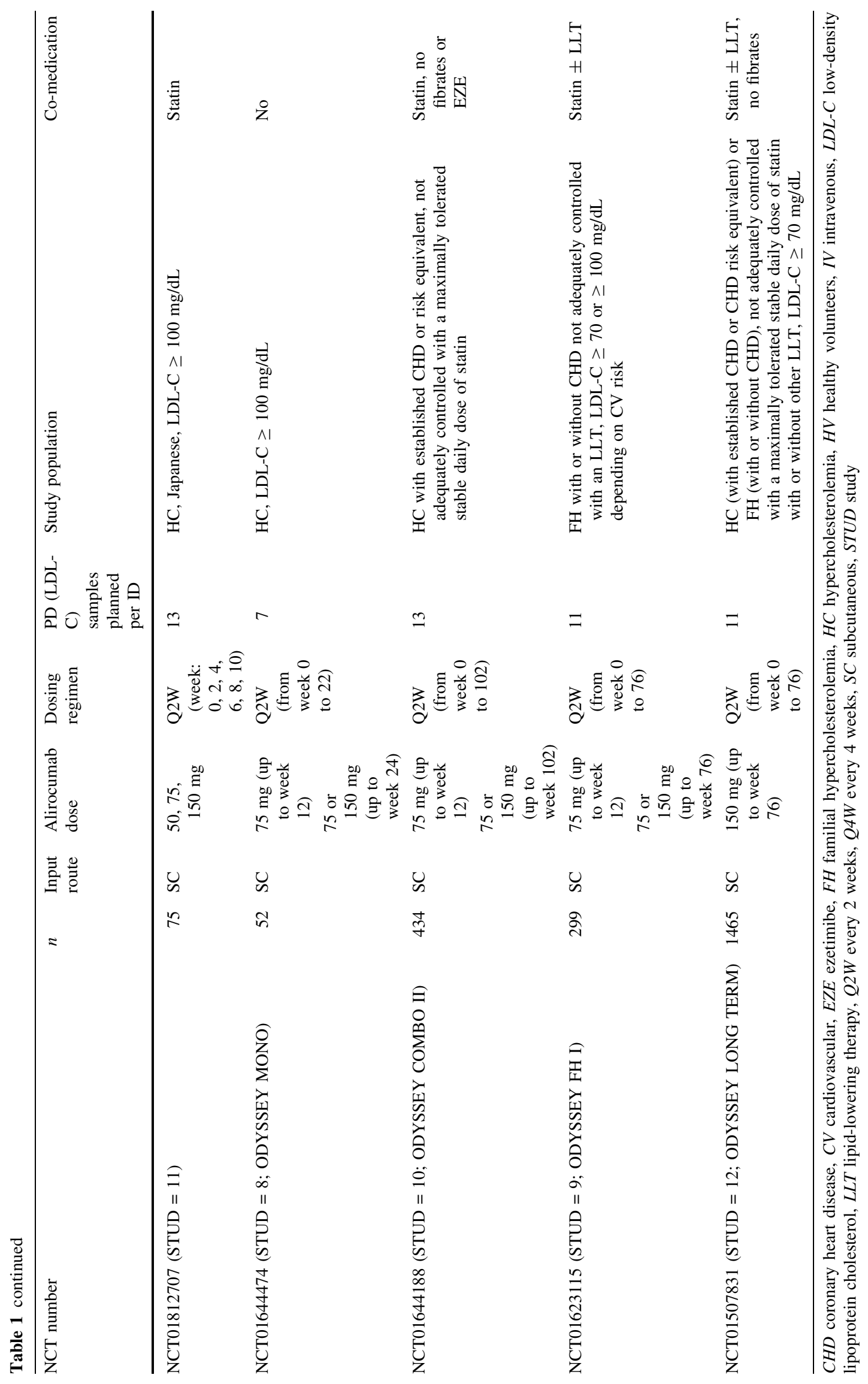


with one degree of freedom; log-likelihood ratio test) in the $\mathrm{OFV}$ ( $\triangle \mathrm{OFV}$ of $\geq 3.84$ units) when introduced into the model were retained. Finally, each covariate was removed in turn (backward deletion) and its impact on OFV was determined; a $\triangle \mathrm{OFV}$ of less than 10.8 , associated with significance of $p<0.001$ (log-likelihood ratio test), led to the exclusion of the covariate from the final population model.

\subsection{Model Verification and Qualification}

Model verification was performed for both the pharmacostatistical model and final model by examination of goodness-of-fit plots and by calculating several quality criteria, such as mean prediction error or average fold error. Qualification of the predictive ability of the final PopPK/ PD model was performed using visual predictive check and bootstrap methods.

\subsection{Computation of Individual Pharmacodynamic Parameters}

Following qualification of the PopPK/PD model, the PD parameters were used to derive mean and median values for $\triangle \mathrm{LDL}-\mathrm{C}_{\max }$ (maximum decrease in LDL-C from baseline) and $\triangle \mathrm{LDL}-\mathrm{C}_{\text {trough }}$ (difference in LDL-C between baseline and value before next alirocumab dose). Derived parameters (such as $\Delta \mathrm{LDL}-\mathrm{C}_{\max }$ and $\Delta \mathrm{LDL}-\mathrm{C}_{\text {trough }}$ ) were calculated for patients in phase III studies (receiving 75 and $150 \mathrm{mg}$ Q2W) in the dosing interval on weeks 10-12 (before possible dose increase) and weeks 22-24 (primary endpoint in all phase III studies). To do this, a rich virtual sampling schedule was added following the administrations of interest, and the corresponding individual LDL-C vs. time curves were predicted. The virtual sampling schedule was identical for all individuals receiving the same dosing regimen: patients were virtually sampled every $12 \mathrm{~h}$ after alirocumab administration up until $336 \mathrm{~h}$ (day 14). Additional descriptive statistics for $\Delta L D L-C_{\max }$ and $\Delta L D L-$ $\mathrm{C}_{\text {trough }}$ were provided as a function of the following covariates: sex, age, body weight, body mass index, race, free and total baseline PCSK9, renal function, and statin co-administration.

\section{Results}

\subsection{Pharmacostatistical Population Pharmacokinetic/Pharmacodynamic Model}

A schematic of the turnover model, or indirect response model, developed to link alirocumab concentrations to LDL-C levels, is shown in Fig. 1. The pharmacostatistical

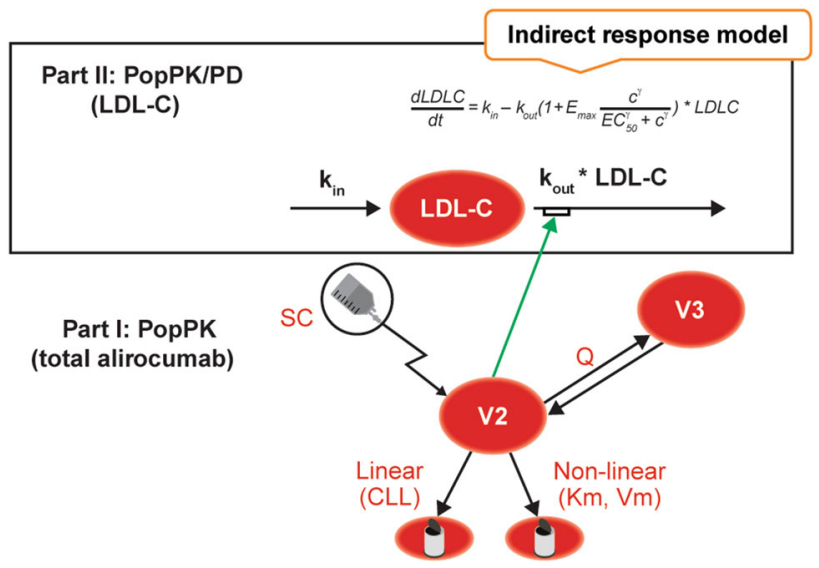

Fig. 1 Schematic of the pharmacostatistical population pharmacokinetic/pharmacodynamic (PopPK/PD) model. An indirect response model was developed to link total alirocumab concentrations (Part I) [27] to low-density lipoprotein cholesterol (LDL-C) levels (Part II). The PopPK/PD model was parameterized with a first-order rate constant for loss of response $\left(K_{\text {out }}\right)$, a maximum drug-induced effect $\left(E_{\max }\right)$, an alirocumab concentration inducing $50 \%$ of $E_{\max }\left(\mathrm{EC}_{50}\right)$, and a Hill coefficient $(\gamma)$. As described in Part 1, the population pharmacokinetic model was developed using a Michaelis-Menten approximation of a target-mediated drug disposition model, characterized by central and peripheral compartment volumes ( $V 2$ and $V 3$, respectively), inter-compartmental clearance $(Q)$, linear elimination clearance (CLL), and a non-linear process represented by two Michaelis-Menten parameters $(\mathrm{Km}, \mathrm{Vm})$, associated with the subcutaneous (SC) administration of alirocumab

PopPK/PD model was parameterized with a first-order rate constant for loss of response $\left(K_{\text {out }}\right)$, a maximum drug-induced effect $\left(E_{\max }\right)$, an alirocumab concentration inducing $50 \%$ of $E_{\max }\left(\mathrm{EC}_{50}\right)$, and a Hill coefficient $(\gamma)$. The interindividual (residual) variability was modeled through an exponential error model and estimated for all PD model parameters. A combined (additive + proportional) error model was used to model the residual variability. The PopPK/PD parameters of the pharmacostatistical model are summarized in Table 2. The impact of any error or bias linked to the bioanalysis (such as LDL-C measurements) is reflected in the estimation of the residual variability, in addition to the impact of adherence issues, and errors linked to the sample collection. In the current pharmacokinetic/pharmacodynamic analysis, there is a combined residual model with an additive error of $5.15 \mathrm{mg} / \mathrm{dL}$ and a proportional error of $22.3 \%$, which is within acceptable limits (Table 2).

\subsection{Covariate Screening}

Potential co-linearity between continuous covariates was identified by a scatterplot matrix, as shown in Fig. 1 of the ESM. The final PopPK/PD model included a total of ten covariates that were retained to explain between-subject variability: six covariates for $E_{\max }$ [sex, age, weight, free 
Table 2 Population pharmacokinetic/pharmacodynmaic parameters before (pharmacostatistical model) and after (final model) inclusion of covariates, compared with bootstrap results

\begin{tabular}{|c|c|c|c|c|c|c|c|}
\hline \multirow[t]{2}{*}{ Parameter } & \multicolumn{2}{|c|}{$\begin{array}{l}\text { Pharmacostatistical } \\
\text { model }\end{array}$} & \multicolumn{3}{|c|}{ Final model with covariates } & \multicolumn{2}{|c|}{ Bootstrap estimates } \\
\hline & $\begin{array}{l}\text { Estimate }(\mathrm{CV} \\
\%)\end{array}$ & $\begin{array}{l}\% \\
\mathrm{RSE}\end{array}$ & $\begin{array}{l}\text { Estimate }(\mathrm{CV} \\
\%)\end{array}$ & $\begin{array}{l}\% \\
\text { RSE }\end{array}$ & $\begin{array}{l}95 \% \text { CI (shrinkage } \\
\%)\end{array}$ & Median & $95 \% \mathrm{CI}$ \\
\hline Typical value of $K_{\text {out }}\left(\mathrm{h}^{-1}\right)$ & 0.00522 & 4.77 & 0.00395 & 6.74 & $0.00342-0.00449$ & 0.00396 & $0.00343-0.00454$ \\
\hline Effect of DISST on $K_{\text {out }}^{\text {a }}$ & NA & NA & 0.00997 & 5.73 & $0.00883-0.0111$ & 0.00978 & $0.00768-0.0132$ \\
\hline Typical value of $\mathrm{EC}_{50}(\mathrm{mg} / \mathrm{L})$ & 3.95 & 2.74 & 1.44 & 13.0 & $1.07-1.82$ & 1.40 & $0.890-2.34$ \\
\hline $\begin{array}{l}\text { Effect of TBSPCSK9 on } \\
\text { EC }_{50} \text { b }\end{array}$ & NA & NA & 0.00219 & 12.4 & $0.00164-0.00273$ & 0.00225 & $0.000540-0.00305$ \\
\hline $\begin{array}{l}\text { Effect of HDSTATIN on } \\
\mathrm{EC}_{50} \mathrm{~b}\end{array}$ & NA & NA & 1.21 & 3.89 & $1.11-1.30$ & 1.20 & $1.09-1.34$ \\
\hline Typical value of $E_{\max }$ & 2.72 & 2.07 & 2.43 & 4.07 & $1.63-1.92$ & 2.46 & $2.24-2.91$ \\
\hline Effect of TPCSK9 on $E_{\max }{ }^{c}$ & NA & NA & 0.000331 & 3.69 & $0.000306-0.000355$ & 0.000328 & $0.000266-0.000418$ \\
\hline Effect of SEX on $E_{\max }{ }^{c}$ & NA & NA & 0.703 & 3.71 & $0.651-0.755$ & 0.708 & $0.648-0.773$ \\
\hline Effect of AGE on $E_{\max }{ }^{\mathrm{c}}$ & NA & NA & 0.415 & 15.6 & $0.286-0.545$ & 0.404 & -0.00165 to 0.544 \\
\hline Effect of WEIGHT on $E_{\max }{ }^{c}$ & NA & NA & 0.313 & 26.7 & $0.146-0.480$ & 0.278 & -0.00496 to 0.465 \\
\hline $\begin{array}{l}\text { Effect of FBSPCSK9 on } \\
E_{\max }\end{array}$ & NA & NA & 0.00156 & 19.1 & $0.000965-0.00215$ & 0.00170 & 0.000529-0.00295 \\
\hline Effect of STATIN on $E_{\max }{ }^{c}$ & NA & NA & 0.408 & 20.4 & $0.242-0.575$ & 0.363 & -0.00493 to 0.601 \\
\hline Typical value of $\gamma$ & 2.18 & 4.33 & 1.78 & 4.07 & $1.63-1.92$ & 1.81 & $1.51-2.18$ \\
\hline Effect of FBSPCSK9 on $\gamma^{\mathrm{d}}$ & NA & NA & 0.00340 & 13.3 & $0.00249-0.00430$ & 0.00309 & $0.000601-0.00481$ \\
\hline \multicolumn{8}{|c|}{ Inter-individual variability (CV \%) } \\
\hline$K_{\text {out }}$ & $0.633(79.6)$ & 7.92 & $0.113(33.7)$ & 33.7 & 0. 0369-0.190 (82.4) & 0.136 & $0.000100-0.826$ \\
\hline $\mathrm{EC}_{50}$ & $0.089(29.9)$ & 12.5 & $0.123(35.1)$ & 12.6 & $0.0921-0.154(64.5)$ & 0.115 & $0.0191-0.202$ \\
\hline$E_{\max }$ & $0.491(70.1)$ & 3.13 & $0.420(65.8)$ & 3.01 & $0.395-0.445(11.2)$ & 0.423 & $0.383-0.468$ \\
\hline$\gamma$ & $0.422(65.0)$ & 11.8 & $0.296(54.4)$ & 12.5 & $0.222-0.371(61.3)$ & 0.255 & $0.0416-0.482$ \\
\hline \multicolumn{8}{|l|}{ Residual variability } \\
\hline Additive term (mg/dL) & 5.148 & 2.13 & 5.21 & 2.13 & $4.99-5.43$ & 5.23 & $4.67-5.83$ \\
\hline Proportional term & 0.223 & 0.57 & 0.224 & 0.59 & $0.221-0.227$ & 0.223 & $0.212-0.236$ \\
\hline
\end{tabular}

$C I$ confidence interval, $C V$ coefficient of variation, DISST status of healthy volunteer or patient, $E C_{50}$ alirocumab concentration inducing $50 \%$ of $E_{\max }, E_{\max }$ maximum drug-induced effect, FBSPCSK9 free baseline value of PCSK9, HDSTATIN co-administration of high-dose statin, $K_{\text {out }}$ firstorder rate constant for loss of response, NA not applicable, RSE relative standard error $(100 \% \times$ SE/estimate), SE standard error, TBSPCSK 9 total baseline value of PCSK9, $\gamma$ Hill coefficient

${ }^{\text {a }}$ The expression of $\mathrm{K}_{\text {out }}$ including the covariates effects is: $K_{\text {out }}=0.00395 *(1-$ DISST $)+0.00997 *$ DISST, where DISST was coded 0 if healthy volunteer and 1 if patient

${ }^{\mathrm{b}}$ The expression of $\mathrm{EC}_{50}$ including the covariates effects is: $\mathrm{EC}_{50}=(1.44+0.00219 *$ TBSPCSK9 $) * 1.21 * *$ HDSTATIN, where TBSPCSK9 is the PCSK9 serum level at baseline in the total data set and HDSTATIN was coded 1 for co-administration of rosuvastatin ( $\geq 20 \mathrm{mg} / \mathrm{d}$ ) or atorvastatin $(\geq 40 \mathrm{mg} / \mathrm{d})$ and was coded 0 for no statin or a lower dose of statin

${ }^{\mathrm{c}}$ The expression $\mathrm{E}_{\max }$ including the covariates is: $\mathrm{E}_{\max }=(((2.43+0.000331 *(\mathrm{TPCSK} 9-3340)) * 0.703 * * \mathrm{SEX}) *(\mathrm{AGE} / 60) * * 0.415 *$ $($ WEIGHT/82.5) $* * 0.313)+0.00156 *($ FBSPCSK9 -265$)+0.408 *$ STATIN, where TPCSK9 is the time-varying PCSK9 serum level and 3340 is the median in the total data set; 60 is the median value of AGE in the total data set; 82.5 is the median value of WEIGHT in the total data set; FBSPCSK 9 is the free baseline value of PCSK9 and 265 is the median value in the total data set; STATIN was coded 0 if alirocumab was given alone and coded 1 if co-administered with a statin; and SEX was coded 0 for male and coded 1 for female

${ }^{\mathrm{d}}$ The expression of $\gamma$ including the covariate is: $\gamma=1.78+0.00340 *($ FBSPCSK9 -265$)$

baseline PCSK9 (FBSPCSK9), total time-varying PCSK9 levels (TPCSK9) and concomitant statin administration (STATIN)], two for $\mathrm{EC}_{50}$ [total baseline PCSK9 (TBSPCSK9) and co-administration of high-dose statins (rosuvastatin $\geq 20 \mathrm{mg} /$ day or atorvastatin $\geq 40 \mathrm{mg} /$ day; HDSTATIN)], one for $\gamma$ (FBSPCSK9), and one for $K_{\text {out }}$ [status of healthy volunteer or patient (DISST)]. Using backward deletion to assess the relevance of the covariates selected during the model building process to the final population model did not result in exclusion of any covariate from the final PK/PD model. The relationships between the PD parameters and relevant covariates were described according to the following equations: 


$$
\begin{aligned}
E_{\max }= & (((2.43+0.0003331 \times(\text { TPCSK9 }-3340)) \\
& \left.\left.\times 0.703^{\mathrm{SEX}}\right) \times\left(\frac{\mathrm{AGE}}{60}\right)^{0.415} \times\left(\frac{\text { WEIGHT }}{82.5}\right)^{0.313}\right) \\
& +0.00156 \times(\text { FBSPCSK } 9-265)+0.408 \times \text { STATIN }
\end{aligned}
$$

$$
\mathrm{EC}_{50}=(1.44+0.00219 \times \text { TBSPCSK9 }) \times 1.21^{\text {HDSTATIN }}
$$$$
K_{\text {out }}=0.00395 \times(1-\text { DISST })+0.00997 \times \text { DISST }
$$$$
\gamma=1.78+0.00340 \times(\text { FBSPCSK } 9-265)
$$

The final PopPK/PD parameters are summarized in Table 2, together with those before covariate inclusion, for comparison. The impact of the covariates on each model parameter is shown in Fig. 2 of the ESM.

\subsection{Verification of the Final Population Pharmacokinetic/Pharmacodynamic Model}

The standard errors of the PopPK/PD parameter estimates (\% relative standard error) were sufficiently small such that the 95\% confidence intervals did not include zero (Table 2). The main quality criteria computed for the comparison of population (PRED) and individual (IPRED) vs. observed (OBS) values of LDL-C for the final model are given in Table 3. The mean prediction error was very low but significantly different from zero for both PRED and IPRED predicted LDL-C levels vs. OBS values, representing 4.04 and $1.51 \%$ of the mean OBS for PRED and IPRED, respectively (Table 3). Average fold error values were 1.44 and 1.21, and correlation coefficients were 0.783 and 0.930 , for PRED and IPRED vs. OBS, respectively (Table 3). No important systematic deviation or major bias was noted in any of the goodness-of-fit plots [conditional weighted residuals and individual weighted residuals vs. PRED and IPRED, Fig. 2; PRED and IPRED vs. OBS, Fig. 2 (logarithmic scale, Fig. 3 of the ESM)].

\subsection{Qualification of the Final Population Pharmacokinetic/Pharmacodynamic Model}

The robustness of the final model and accuracy of the parameter estimates were assessed using an un-stratified non-parametric bootstrap method. Of the 1243 bootstraps launched, 539 runs were successful $(43.4 \%)$; the median values obtained from the bootstrap are compared to the parameters of both the pharmacostatistical and final models in Table 2. All the fixed-effect parameters as well as the inter-individual terms estimated with the final model are within the 95\% confidence interval computed from the bootstrap, which suggested an overall good qualification of the final model (Table 2).

For the effect of age and weight on $E_{\max }$, the distribution was not uniform as two clusters are present. During the bootstrap process, the new data sets were generated by sampling individuals with replacement from the original dataset, and fitting the model to each new dataset. Thus, given the large heterogeneity of the data, the final parameters' estimates might be impacted by the number of patients from each study and the associated covariates included in each generated data set. The results of the visual predictive check showed that the vast majority of the observed LDL-C values were within the 5th and 95th predicted percentiles (Fig. 3), qualifying the good predictive performance of the PopPK/PD model. The inter-individual variability was estimated for all the pharmacodynamic parameters: $33.7 \%$ for $K_{\text {out }}, 35.1 \%$ for $\mathrm{EC}_{50}, 64.8 \%$ for $E_{\max }$, and $54.4 \%$ for $\gamma$.

\subsection{Impact of Covariates on Model Parameters}

The relationship between $\mathrm{E}_{\max }$ and the six covariates included in the final PopPK/PD model was evaluated for the extreme values based on the combination of sex and statin covariates and assuming median values for the other continuous covariates. The impact of covariates on $E_{\max }$ was quite variable. Time-varying total PCSK9 resulted in $E_{\max }$ values of $1.05-1.89$ for the 5 th percentile $(491 \mathrm{ng} / \mathrm{mL})$ and of $2.41-3.83$ for the 95th percentile $(6340 \mathrm{ng} / \mathrm{mL})$ with an impact of a $93-130 \%$ increase in $E_{\max }$ (between 5th and 95th percentiles; calculations tabulated in Table 3 of the ESM). Free baseline PCSK9 resulted in $E_{\max }$ values of 1.56-2.62 for the 5th percentile $(126 \mathrm{ng} / \mathrm{mL})$ and of $1.97-3.21$ for the 95th percentile $(501 \mathrm{ng} / \mathrm{mL})$ with an impact of a 20.9 to $26.4 \%$ increase. Weight resulted in $E_{\max }$ values of $1.52-2.58$ for the 5th percentile $(58.1 \mathrm{~kg})$ and of $1.91-3.12$ for the 95th

Table 3 Comparison of population (PRED) and individual (IPRED) predicted low-density lipoprotein cholesterol levels with observed (OBS) low-density lipoprotein cholesterol values in the final population pharmacokinetic/pharmacodynamic model

\begin{tabular}{lll}
\hline Quality criteria & PRED vs. OBS (mg/dL) & IPRED vs. OBS (mg/dL) \\
\hline Mean prediction error [95\% CI] (\% mean OBS) & $2.44[2.02-2.87](4.04)$ & 0.919 [0.670-1.17] (1.51) \\
Correlation $(r)$; slope; intercept & $0.783 ; 0.644 ; 19.1$ & $0.930 ; 0.850 ; 8.17$ \\
Average fold error & 1.44 & 1.21 \\
\hline
\end{tabular}

CI confidence interval 

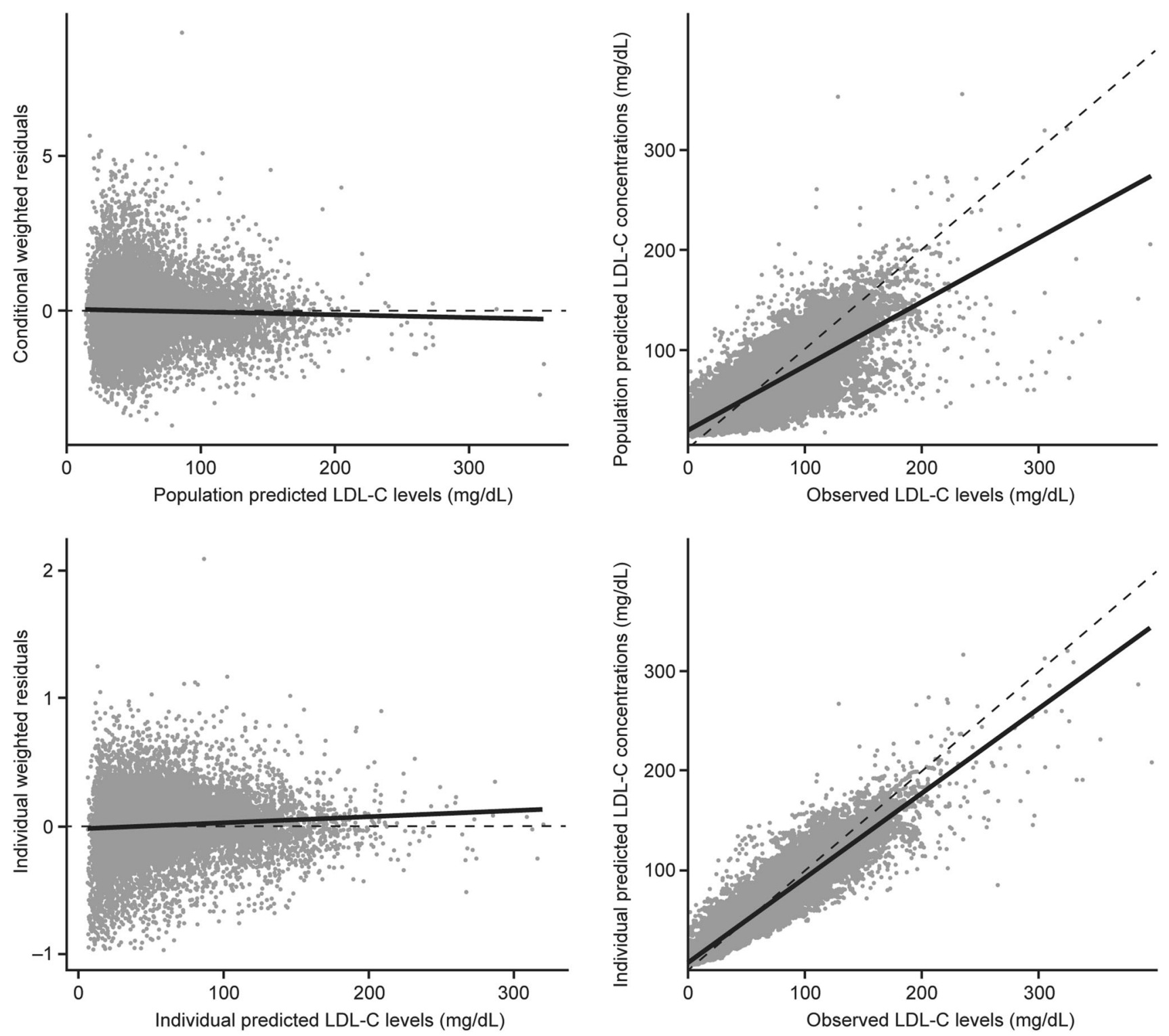

Fig. 2 Relationship between population (top panels) or individual (bottom panels) predicted levels of low-density lipoprotein cholesterol (LDL-C) and conditional or individual weighted residuals (left

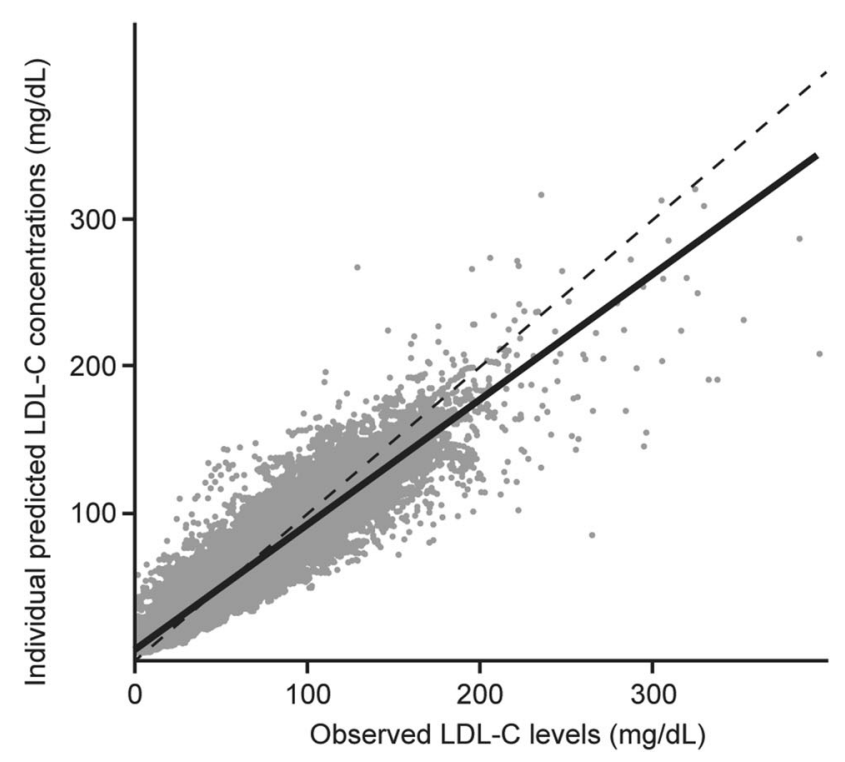

panels) and observed levels of LDL-C (right panels) after covariate inclusion. The tendency line is shown as a solid black line

percentile $(119 \mathrm{~kg})$ with an impact of a $19.8-25.2 \%$ increase. Sex was associated with a $29.7 \%$ decrease in $E_{\max }$ values for female vs. male subjects. Age resulted in $E_{\max }$ values of $1.40-2.40$ for the 5 th percentile ( 37 years) and values of 1.87-3.07 for the 95th percentile ( 75 years) with an impact of a $26.4-34.1 \%$ increase. It should be noted that the estimated impact of sex and age was independent of body weight. Finally, concomitant statin therapy was associated with a $16.8 \%$ increase in $E_{\max }$.

The relationship between $\mathrm{EC}_{50}$, total baseline PCSK9, and high-dose statin resulted in $\mathrm{EC}_{50}$ values of $2.22 \mathrm{mg} / \mathrm{L}$ (without a high-dose statin) to $2.67 \mathrm{mg} / \mathrm{L}$ (with a high- dose statin) for the 5 th percentile $(355 \mathrm{ng} / \mathrm{mL})$ and of $3.91 \mathrm{mg} / \mathrm{L}$ (without a high-dose statin) to $4.71 \mathrm{mg} / \mathrm{L}$ (with a high-dose statin) for the 95th percentile of total baseline PCSK9 $(1130 \mathrm{ng} / \mathrm{mL})$; an impact of a $76.5 \%$ increase of $\mathrm{EC}_{50}$ from the 5th to 95 th percentiles of total baseline PCSK9 and an increase of $20.6 \%$ when a high-dose statin was co-administered compared with no high-dose statin co-medication.

The relationship between the Hill coefficient, $\gamma$, and free baseline PCSK9 values resulted in $\gamma$ values of 1.31 , 1.78 , and 2.58 for the 5 th $(126 \mathrm{ng} / \mathrm{mL})$, 50th $(265 \mathrm{ng} /$ $\mathrm{mL})$, and 95 th $(501 \mathrm{ng} / \mathrm{mL})$ percentiles of free PCSK9 at 


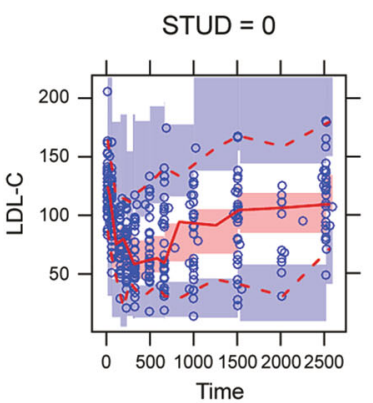

STUD $=4$

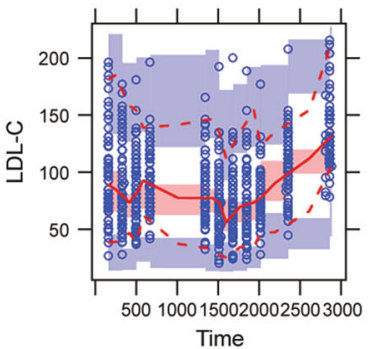

STUD $=1$

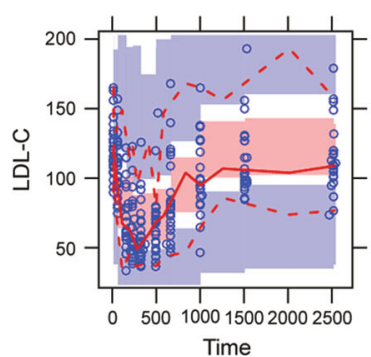

STUD $=5$

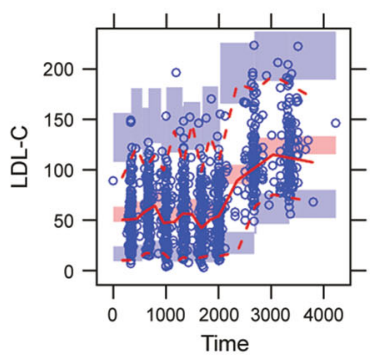

STUD $=2$

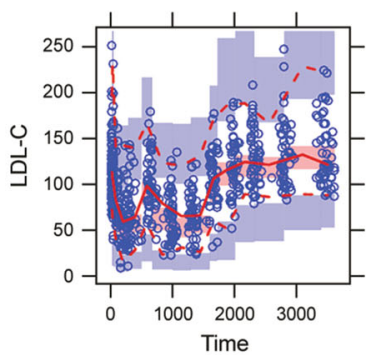

STUD $=6$

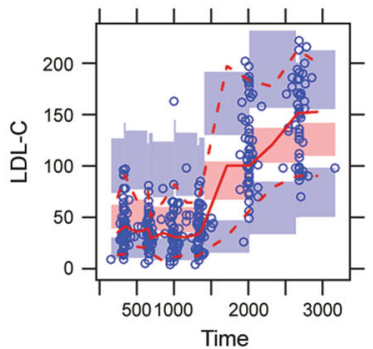

STUD $=3$

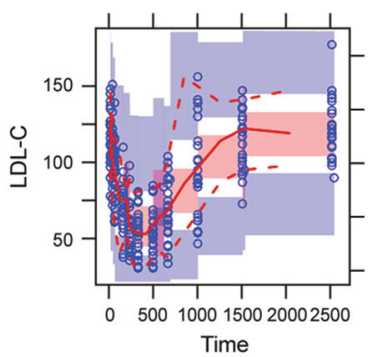

STUD $=7$

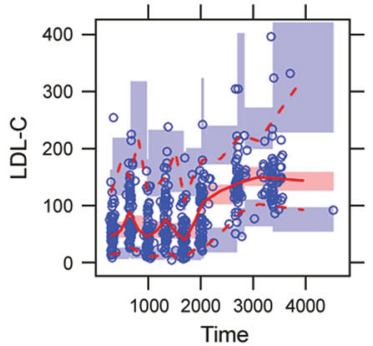

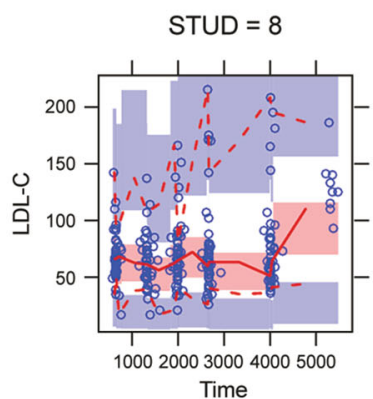
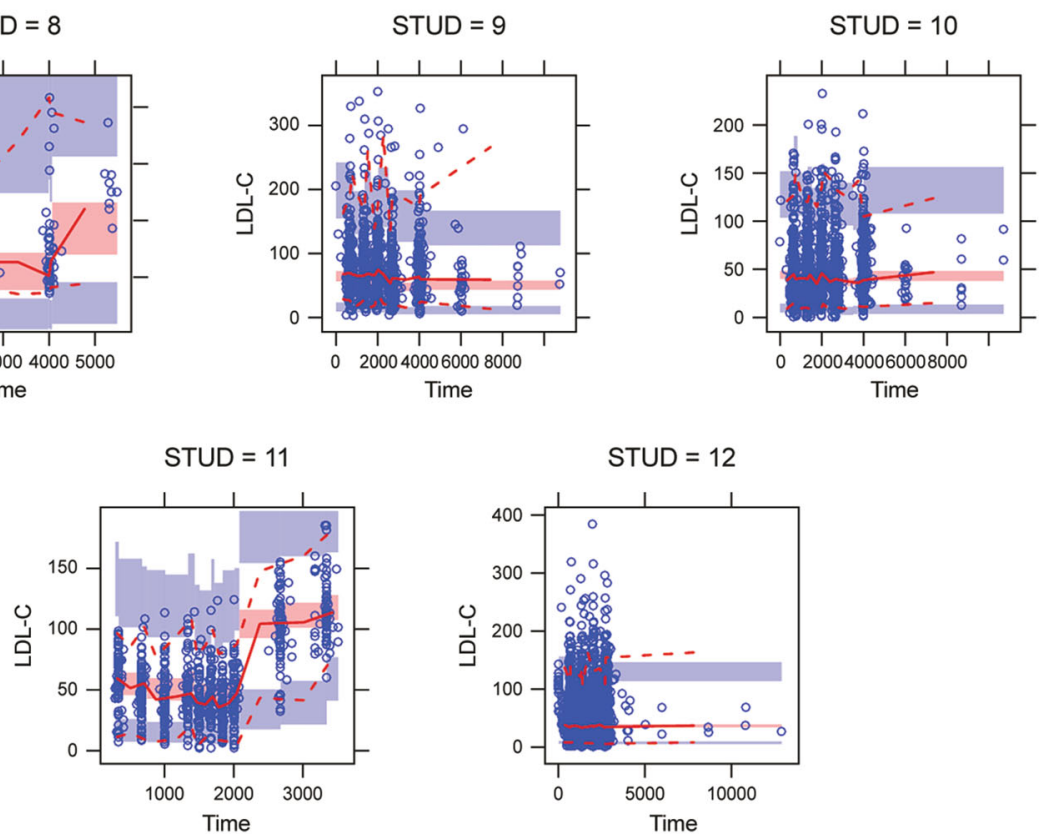

Fig. 3 Visual predictive check results [low-density lipoprotein cholesterol (LDL-C) vs. time] for each of the 13 studies. Dark blue dots indicate observations; solid red line indicates the median of observations; solid dashed lines indicate the 5th and 95th percentiles

baseline, with an impact of $97.4 \%$ on $\gamma$ from the 5th to 95th percentiles of free baseline PCSK9. The relationship between $K_{\text {out }}$ and disease state resulted in a $K_{\text {out }}$ value of $0.00395 \mathrm{~h}^{-1}$ for healthy volunteers and $0.00997 \mathrm{~h}^{-1}$ for patients, with an impact of a 2.52 -fold increase in $K_{\text {out }}$ in patients compared with healthy volunteers.

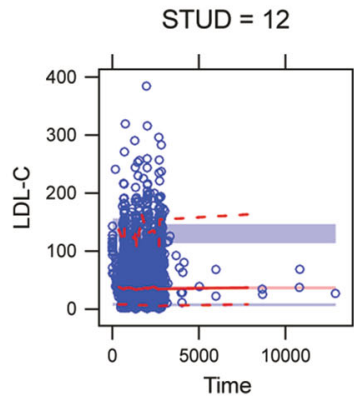

of observations; pink and blue areas indicate confidence intervals of the median and 5th and 95th percentiles of predictions, respectively. STUD study

\subsection{Impact of Covariates on Low-Density Lipoprotein Cholesterol: Derived Pharmacodynamic Parameters}

Following development and validation of the PopPK/PD model, the model parameters were used to derive individual exposures $\left(\Delta \mathrm{LDL}-\mathrm{C}_{\max }\right.$ and $\Delta \mathrm{LDL}-\mathrm{C}_{\text {trough }}$ in the dosing 
Table 4 Summary of derived values for maximum percentage change from baseline in lowdensity lipoprotein cholesterol $\left(\Delta\right.$ LDL- $\left.\mathrm{C}_{\max }\right)$ and percentage change from baseline in LDL-C at pre-dose $\left(\Delta \mathrm{LDL}-\mathrm{C}_{\text {trough }}\right)$ on weeks $10-12$ and $22-24$ for phase III subjects

\begin{tabular}{|c|c|c|c|c|}
\hline \multirow[t]{3}{*}{ Descriptive statistics } & \multicolumn{4}{|c|}{ Derived PD parameters $(\%)$ computed on week $10-12$} \\
\hline & \multicolumn{2}{|c|}{75 -mg dose $(n=754)$} & \multicolumn{2}{|c|}{150 -mg dose $(n=1407)$} \\
\hline & $\Delta \mathrm{LDL}-\mathrm{C}_{\max }$ & $\Delta \mathrm{LDL}-\mathrm{C}_{\text {trough }}$ & $\Delta \mathrm{LDL}-\mathrm{C}_{\max }$ & $\Delta \mathrm{LDL}-\mathrm{C}_{\text {trough }}$ \\
\hline Mean & 62.1 & 54.6 & 71.5 & 68.9 \\
\hline $\mathrm{CV}$ & 23.9 & 29.2 & 18.7 & 21.0 \\
\hline SD & 14.8 & 15.9 & 13.4 & 14.5 \\
\hline Minimum & 0 & 0 & 16.8 & 8.40 \\
\hline Median & 63.6 & 56.4 & 74.4 & 70.8 \\
\hline Maximum & 92.4 & 92.4 & 94.8 & 93.6 \\
\hline [5th; 95th] percentiles & {$[32.4 ; 82.8]$} & {$[24.0 ; 78.0]$} & {$[45.6 ; 88.8]$} & {$[40.8 ; 87.6]$} \\
\hline \multirow[t]{3}{*}{ Descriptive statistics } & \multicolumn{4}{|c|}{ Derived PD parameters $(\%)$ computed on week $22-24^{\mathrm{a}}$} \\
\hline & \multicolumn{2}{|c|}{$75 \mathrm{mg}$ dose $(\mathrm{n}=518)$} & \multicolumn{2}{|c|}{$150 \mathrm{mg}$ dose $(\mathrm{n}=1541)$} \\
\hline & $\Delta \mathrm{LDL}-\mathrm{C}_{\max }$ & $\Delta \mathrm{LDL}-\mathrm{C}_{\text {trough }}$ & $\Delta \mathrm{LDL}-\mathrm{C}_{\max }$ & $\Delta \mathrm{LDL}-\mathrm{C}_{\text {trough }}$ \\
\hline Mean & 67.6 & 60.2 & 70.4 & 67.4 \\
\hline $\mathrm{CV}$ & 16.7 & 21.6 & 20.1 & 22.5 \\
\hline SD & 11.3 & 13.0 & 14.1 & 15.2 \\
\hline Minimum & 15.6 & 7.20 & 6.00 & 4.80 \\
\hline Median & 68.4 & 61.2 & 73.2 & 69.6 \\
\hline Maximum & 92.4 & 91.2 & 93.6 & 93.6 \\
\hline [5th; 95th] percentiles & {$[49.2 ; 85.2]$} & {$[36.0 ; 80.4]$} & {$[43.2 ; 88.8]$} & {$[38.4 ; 87.6]$} \\
\hline
\end{tabular}

$C V$ coefficient of variation, $P D$ pharmacodynamic, $S D$ standard deviation

${ }^{a}$ These statistics take into account patients who had an alirocumab dose increase from 75 to $150 \mathrm{mg}$ at week 12 in MONO, COMBO II, and FH I studies. As there was no dose increase in LONG TERM, and as the steady state was achieved at week 13, the derived PD parameters for MONO, COMBO II, and FH I studies computed by simulation on week 22-24 were pooled with the derived PD parameters computed by simulation between week 13 and week 30 for LONG TERM interval) for phase III patients. Descriptive statistics of derived values for $\Delta \mathrm{LDL}-\mathrm{C}_{\max }$ and $\Delta \mathrm{LDL}-\mathrm{C}_{\text {trough }}$ at weeks 10-12 and 22-24 (after the 6th and 11th alirocumab dose, respectively) are summarized in Table 4 , per dose $(75 \mathrm{mg}$ Q2W or $150 \mathrm{mg}$ Q2W) for study participants. At weeks 10-12, median $\Delta$ LDL- $C_{\max }$ was 63.6 and $74.4 \%$, and median $\Delta$ LDL- $C_{\text {trough }}$ was 56.4 and $70.8 \%$ for the $75-\mathrm{mg}$ and 150-mg Q2W dose, respectively. At weeks 22-24, median $\Delta$ LDL- $\mathrm{C}_{\max }$ was 68.4 and $73.2 \%$, and median $\Delta \mathrm{LDL}-\mathrm{C}_{\text {trough }}$ was 61.2 and $69.6 \%$ for the $75-\mathrm{mg}$ and 150-mg dose, respectively. Box plots illustrating $\triangle$ LDL$\mathrm{C}_{\text {trough }}$ and $\Delta \mathrm{LDL}-\mathrm{C}_{\max }$ per dose and per covariate are shown in Fig. 4 . When comparing the $\Delta$ LDL- $\mathrm{C}_{\text {trough }}$ measurements at weeks $10-12$ and weeks $22-24$ for the 75-mg Q2W dose, it is important to note that the weeks 10-12 measurement includes all patients starting on the 75-mg Q2W dose, whereas the weeks 22-24 measurement only includes patients who did not need a dose increase to $150 \mathrm{mg}$ Q2W.

\section{Discussion}

Using data from 13 phase I/II/III studies in patients with hypercholesterolemia and healthy subjects, we developed and qualified an indirect PopPK/PD response model to describe the relationship between alirocumab concentrations and LDL-C levels. This 'type IV' indirect response model was selected based on the mechanism of action of alirocumab, which leads to an increase in LDL-C elimination. The pharmacostatistical model was parameterized with a first-order rate constant for the loss of response $\left(K_{\text {out }}\right)$, a maximum drug-induced effect $\left(E_{\max }\right)$, the alirocumab concentration at $50 \%$ of maximal effect $\left(\mathrm{EC}_{50}\right)$, and a Hill coefficient $(\gamma)$. The potential sources of betweensubject variability on the pharmacodynamic parameters were investigated; ten covariates were included in the final model, on $E_{\max }$ (total time-varying PCSK9, sex, age, weight, free baseline PCSK9, and co-administration of statins), $\mathrm{EC}_{50}$ (total baseline PCSK9 and high-dose statin), $K_{\text {out }}$ (disease state), or $\gamma$ (free baseline PCSK9). 

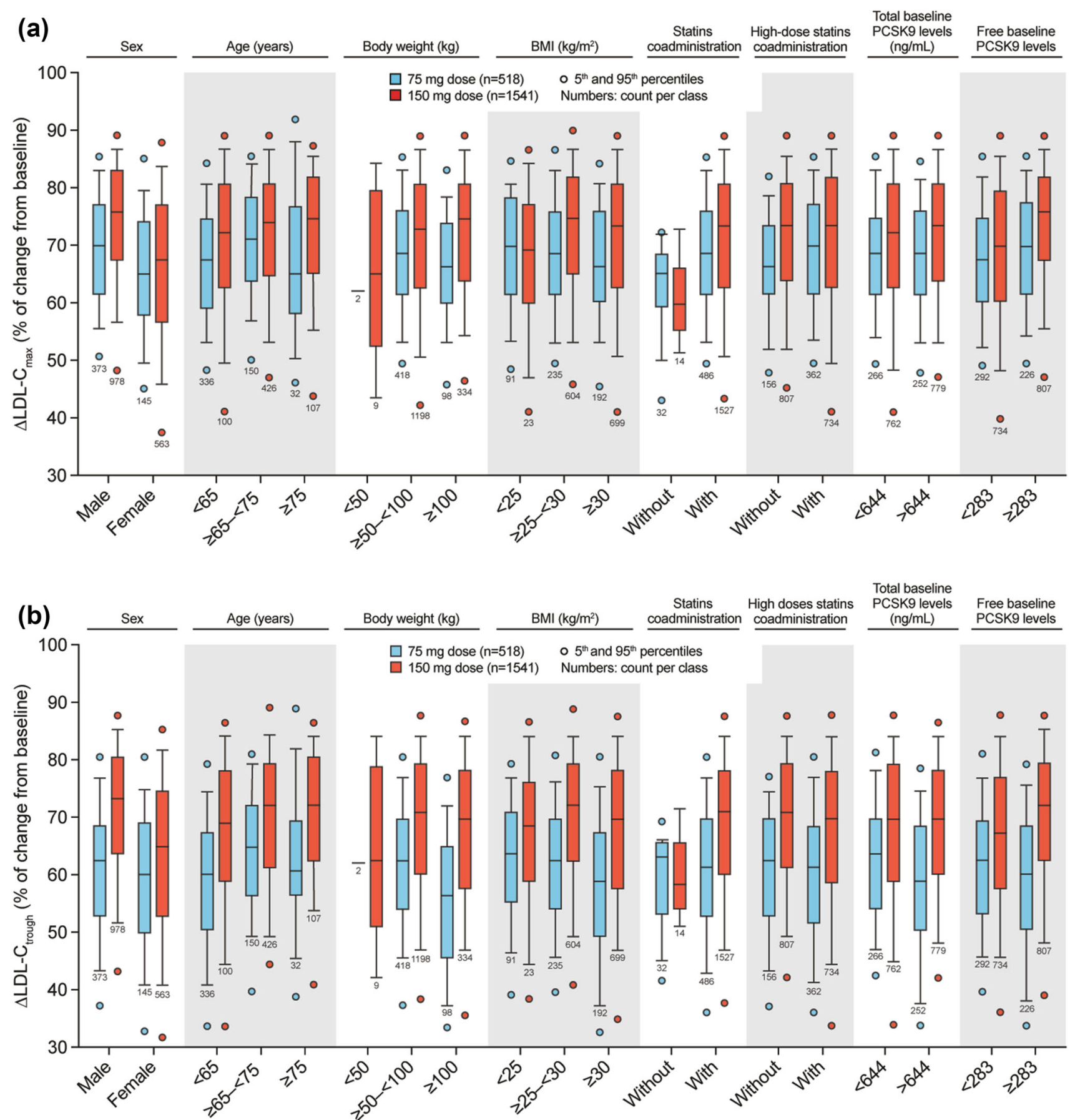

Fig. 4 Box plot of a the maximum percentage change from baseline in low-density lipoprotein cholesterol $\left(\Delta \mathrm{LDL}-\mathrm{C}_{\max }\right)$ and $\mathbf{b}$ the percentage change from baseline LDL-C at pre-dose $\left(\Delta\right.$ LDL- $\left.\mathrm{C}_{\text {trough }}\right)$

The 95th percentile of the time-varying total PCSK9 covariate was found to be associated with approximately a two-fold higher $E_{\max }$ value compared with the 5th percentile; this relationship is consistent with the inhibitory mechanism of action of alirocumab on PCSK9, with higher levels of PCSK9 leading to a greater drug-induced effect. Compared with time-varying total PCSK9, baseline free at week 22-24 in phase III patients as a function of covariates and alirocumab dose. BMI body mass index, PCSK 9 proprotein convertase subtilisin/kexin type 9

PCSK9 resulted in a lower impact on $E_{\max }$ but was still associated with an increase of 20.9-26.4\% from the 5th to 95th percentile. The co-administration of statins, known to upregulate PCSK9 expression [38, 39], led to a $16.8 \%$ increase in $E_{\max }$ even when free baseline PCSK9 was already accounted for in the model. This can be explained by a synergistic effect between the different mechanisms of 
action of statins and alirocumab; alirocumab counteracts statin-induced up-regulation of PCSK9 expression by binding to PCSK9, thereby enhancing the effect of statins [38, 40-42].

Sex was found to influence the LDL-C-lowering effect, with female individuals having $29.7 \%$ lower $E_{\max }$ values than male individuals; this is consistent with a retrospective analysis including 1378 Caucasians (661 were female and 717 were male) with dyslipidemia receiving lipid-lowering therapies, which reported a $28.8 \%$ lower change in LDL-C from baseline in female individuals compared with male individuals [43].

However, despite a slightly smaller degree of LDL-Clowering observed in women compared with men, women have been shown to derive a similar cardiovascular benefit as men following treatment with lipid-lowering therapies [44]. A significant increase of approximately $30 \%$ in $E_{\max }$ between the 5th and 95th percentiles of age (37 and 75 years, respectively) was observed, again consistent with previously published findings that show older patients derive more benefit from lipid-lowering therapies [45]. The relationship of age and sex with $E_{\max }$ values, regardless of body weight, can be also partly explained by the commonly held view that physiological changes in hormone levels, such as loss of endogenous estrogen after menopause for women, influence circulating PCSK9 levels [46]. Furthermore, results of a recent quantitative systems pharmacology modeling study led the authors to hypothesize that the difference in cholesterol metabolism observed between sexes may be explained by estrogen regulation of a potentially less functional form of PCSK9 (form X), with differing efficacy of LDL-R degradation explaining the variability of response between patients [47].

Assessment of the relationship between total baseline PCSK9 and high-dose statin with the $\mathrm{EC}_{50}$ parameter revealed an increase in $\mathrm{EC}_{50}$ with a higher total baseline PCSK9 associated with co-administration of a high-dose statin. Given the mechanism of action of alirocumab and the nature of the indirect response model used, the link between total baseline PCSK9 and $\mathrm{EC}_{50}$ was expected, such that individuals with high baseline values of total PCSK9 require higher alirocumab concentrations to achieve half of the $E_{\max }$ value. The two-fold higher value of $\gamma$ observed from the 5th to the 95th percentile of free baseline PCSK9 (indicating greater cooperativity in ligand binding) may also be attributable to this fact.

Finally, evaluation of the relationship between disease state and $K_{\text {out }}$ revealed an increase in $K_{\text {out }}$ in patients compared with healthy volunteers. It is worth noting that only 150 healthy volunteers from a total of 2799 participants $(5.36 \%)$ were included in the present analysis. In addition, there was considerable co-linearity between disease state and statin co-administration; most of the patients
(2588, $97.7 \%$ of all patients) were co-administered statins while none of the healthy volunteers received concomitant statin therapy. Therefore, the 2.52-fold higher LDL-C elimination in patients compared with healthy volunteers may be explained by the synergistic effect between the different mechanisms of action of statins and alirocumab, as previously mentioned. The $K_{\text {out }}$ values reported here $\left(0.00395\right.$ and $0.00997 \mathrm{~h}^{-1}$ for healthy volunteers and patients, respectively) are consistent with those reported previously; $K_{\text {out }}$ values of 0.012 and $0.00437-0.01458 \mathrm{~h}^{-1}$ were reported for healthy subjects and patients receiving evolocumab or statin therapy, respectively $[48,49]$.

The relationship between the covariates and model parameters exhibited moderate to strong effects; in contrast, the impact of covariates on the primary efficacy endpoint described by $\Delta$ LDL- $\mathrm{C}_{\max }$ and $\Delta$ LDL- $\mathrm{C}_{\text {trough }}$ was quite limited. Covariates with moderate impact on median $\Delta$ LDL- $\mathrm{C}_{\max }$ included sex [median 7.4\% (75-mg Q2W dose) and $12.5 \%$ (150-mg Q2W dose) higher in male individuals vs. female individuals] and statin co-administration [median 5.6\% (75-mg Q2W dose) and 23.2\% (150$\mathrm{mg}$ Q2W dose) higher in patients receiving statins vs. those without statins]. The moderate effect of body weight on $\Delta$ LDL- $\mathrm{C}_{\max }$ (median 6.8\% [75-mg Q2W dose] and $14.8 \%$ [150-mg Q2W dose] higher in patients weighing $\geq 100 \mathrm{~kg}$ vs $<50 \mathrm{~kg}$ ) suggests that the target PCSK9 is saturated with alirocumab irrespective of the weight range. Covariates with low impact on $\Delta$ LDL- $\mathrm{C}_{\max }$ included age [median $3.6 \%$ lower (75 $\mathrm{mg} \mathrm{Q} 2 \mathrm{~W})$ and 3.3\% higher (150 mg Q2W) in patients $\geq 75$ years of age vs $<65$ years], free baseline PCSK9 [median 3.6\% (75 mg Q2W) and 8.6\% (150 mg Q2W) higher in patients with values $\geq 283$ vs $<283 \mathrm{ng} /$ $\mathrm{mL}$ ], and a high-dose statin [median 5.5\% higher (75 and $150 \mathrm{mg}$ Q2W) for patients receiving a high-dose statin vs. those not]. Last, the baseline total PCSK9 had a very limited impact on median $\Delta \mathrm{LDL}-\mathrm{C}_{\max }$ with exactly the same value $(75 \mathrm{mg}$ Q2W) or a $1.7 \%$ increase $(150 \mathrm{mg}$ Q2W) in patients with total baseline PCSK9 $\geq 644$ vs. $<$ $644 \mathrm{ng} / \mathrm{mL}$.

In this PopPK/PD analysis, a Michaelis-Menten approximation of a TMDD model was used to characterize the pharmacokinetic properties of alirocumab, and then the alirocumab concentrations were used to predict the LDL-C levels. In contrast, in a TMDD model with a quasi-steadystate approximation, the minimization is based on the simultaneous fitting of both total PCSK9 and alirocumab concentrations. Consequently, the quality of the estimation is a compromise between the PCSK9 and alirocumab values, both used as dependent variables, which results in a lower quality estimate for alirocumab concentration using a quasi-steady-state approximation compared with a TMDD model using a Michaelis-Menten approximation. A possible enhancement of our model would be to develop a fully 
mechanistic TMDD PopPK/PD model, using a quasisteady-state approximation which we have recently published [26], to describe the mechanistic elimination of alirocumab and its interaction with PCSK9; the aim would be to use the PCSK9 levels rather than alirocumab concentrations as the predictor of the LDL-C-lowering effect. A great advantage of this type of model is the ability to explore the link between the levels of PCSK9 and LDL-C, independently of the alirocumab administered. However, owing to the size of the dataset and the stiffness of the differential equations, it was not possible to qualify such a model at present; further efforts are ongoing to achieve this important though challenging goal.

\section{Conclusions}

This model allowed the characterization of the PopPK/PD properties of alirocumab in its target population and the estimation of individual LDL-C levels and derived PD parameters. Stratification per covariate retained in the model did not show any major difference of the derived PD parameters, irrespective of the population studied (phase I/II or III). Furthermore, as a result of the alirocumab dose increase strategy (where the dose could be increased from 75 to $150 \mathrm{mg}$ Q2W as required), none of the retained covariates had a clinically meaningful impact on LDL-C reduction.

Acknowledgments The authors thank the participants, their families, and all investigators involved in these studies. The following people from the study sponsors reviewed and provided editorial comments on the manuscript: Carol Hudson, Robert Pordy, and A. Thomas DiCioccio (Regeneron Pharmaceuticals, Inc.), and Veronica Lee, Corinne Hanotin, and Michael Howard (Sanofi). These data have been presented at the Population Approach Group Europe congress in Lisbon, Portugal, 2016.

Author contributions All authors were involved with the study design and analysis and interpretation of the data. All authors critically reviewed the manuscript and approved it for submission. The authors take full responsibility for the content of this article.

\section{Compliance with Ethical Standards}

Funding This study was funded by Sanofi and Regeneron Pharmaceuticals, Inc. Medical writing assistance and editorial support were provided by Rachel Dunn, PhD, of Prime (Knutsford, UK), and funded by Sanofi and Regeneron Pharmaceuticals, Inc. Open access publication of this article was sponsored by Sanofi and Regeneron Pharmaceuticals, Inc.

Conflict of interest Xavier Nicolas, Nassim Djebli, Clémence Rauch, Aurélie Brunet, Fabrice Hurbin, Jean-Marie Martinez, and David Fabre are employees of and stockholders in Sanofi.

Ethics approval All procedures performed in studies involving human participants were in accordance with the ethical standards of the institutional and/or national research committee and with the 1964 Helsinki Declaration and its later amendments or comparable ethical standards.

Consent to participate Informed consent was obtained from all individual participants included in the studies. No identifying information for individual patients is included in this article.

Open Access This article is distributed under the terms of the Creative Commons Attribution-NonCommercial 4.0 International License (http://creativecommons.org/licenses/by-nc/4.0/), which permits any noncommercial use, distribution, and reproduction in any medium, provided you give appropriate credit to the original author(s) and the source, provide a link to the Creative Commons license, and indicate if changes were made.

\section{References}

1. Deedwania P, Barter P, Carmena R, et al. Reduction of lowdensity lipoprotein cholesterol in patients with coronary heart disease and metabolic syndrome: analysis of the treating to new targets study. Lancet. 2006;368(9539):919-28.

2. Piepoli MF, Hoes AW, Agewall S, et al. 2016 European guidelines on cardiovascular disease prevention in clinical practice: the Sixth Joint Task Force of the European Society of Cardiology and Other Societies on Cardiovascular Disease Prevention in Clinical Practice (constituted by representatives of 10 societies and by invited experts). Developed with the special contribution of the European Association for Cardiovascular Prevention and Rehabilitation (EACPR). Eur Heart J. 2016;37(29):2315-81.

3. Lloyd-Jones DM, Morris PB, Ballantyne CM, et al. 2016 ACC Expert Consensus Decision Pathway on the role of non-statin therapies for LDL-cholesterol lowering in the management of atherosclerotic cardiovascular disease risk: a report of the American College of Cardiology Task Force on Clinical Expert Consensus documents. J Am Coll Cardiol. 2016;68(1):92-125.

4. Catapano AL, Graham I, De Backer G, et al. 2016 ESC/EAS guidelines for the management of dyslipidaemias: the Task Force for the Management of Dyslipidaemias of the European Society of Cardiology (ESC) and European Atherosclerosis Society (EAS) developed with the special contribution of the European Association for Cardiovascular Prevention and Rehabilitation (EACPR). Eur Heart J. 2016;37(39):2999-3058.

5. Stone NJ, Robinson JG, Lichtenstein AH, et al. 2013 ACC/AHA guideline on the treatment of blood cholesterol to reduce atherosclerotic cardiovascular risk in adults: a report of the American College of Cardiology/American Heart Association Task Force on Practice Guidelines. J Am Coll Cardiol. 2014;63(25 Pt B):2889-934.

6. Guyton JR, Bays HE, Grundy SM, et al. An assessment by the Statin Intolerance Panel: 2014 update. J Clin Lipidol. 2014;8(3 Suppl.):S72-81.

7. Rosenson RS, Baker SK, Jacobson TA, et al. An assessment by the Statin Muscle Safety Task Force: 2014 update. J Clin Lipidol. 2014;8(3 Suppl.):S58-71.

8. Rojas-Fernandez CH, Goldstein LB, Levey AI, et al. An assessment by the Statin Cognitive Safety Task Force: 2014 update. J Clin Lipidol. 2014;8(3 Suppl.):S5-16.

9. Seidah NG, Benjannet S, Wickham L, et al. The secretory proprotein convertase neural apoptosis-regulated convertase 1 (NARC-1): liver regeneration and neuronal differentiation. Proc Natl Acad Sci USA. 2003;100(3):928-33. 
10. Zaid A, Roubtsova A, Essalmani R, et al. Proprotein convertase subtilisin/kexin type 9 (PCSK9): hepatocyte-specific low-density lipoprotein receptor degradation and critical role in mouse liver regeneration. Hepatology. 2008;48(2):646-54.

11. Urban D, Poss J, Bohm M, Laufs U. Targeting the proprotein convertase subtilisin/kexin type 9 for the treatment of dyslipidemia and atherosclerosis. J Am Coll Cardiol. 2013;62(16):1401-8.

12. Lo Surdo P, Bottomley MJ, Calzetta A, et al. Mechanistic implications for LDL receptor degradation from the PCSK9/ LDLR structure at neutral pH. EMBO Rep. 2011;12(12):1300-5.

13. Tveten K, Holla OL, Cameron J, et al. Interaction between the ligand-binding domain of the LDL receptor and the C-terminal domain of PCSK9 is required for PCSK9 to remain bound to the LDL receptor during endosomal acidification. Hum Mol Genet. 2012;21(6):1402-9.

14. McKenney JM. Understanding PCSK9 and anti-PCSK9 therapies. J Clin Lipidol. 2015;9(2):170-86.

15. Cariou B, Le May C, Costet P. Clinical aspects of PCSK9. Atherosclerosis. 2011;216(2):258-65.

16. Regeneron Pharmaceuticals Inc., Sanofi-aventis US LLC. Praluent prescribing information (USA). 2015. http://products.sanofi. us/praluent/praluent.pdf. Accessed 20 Oct 2017.

17. Regeneron Pharmaceuticals Inc., Sanofi-aventis US LLC. Praluent summary of product characteristics (EC). 2015. http://ec. europa.eu/health/documents/community-register/2015/ 20150923132812/anx_132812_en.pdf. Accessed 20 Oct 2017.

18. Kastelein JJ, Ginsberg HN, Langslet G, et al. ODYSSEY FH I and FH II: 78 week results with alirocumab treatment in 735 patients with heterozygous familial hypercholesterolaemia. Eur Heart J. 2015;2015(36):2996-3003.

19. Robinson JG, Farnier M, Krempf M, et al. Efficacy and safety of alirocumab in reducing lipids and cardiovascular events. N Engl J Med. 2015;372(16):1489-99.

20. Bays H, Gaudet D, Weiss R, et al. Alirocumab as add-on to atorvastatin versus other lipid treatment strategies: ODYSSEY OPTIONS I randomized trial. J Clin Endocrinol Metab. 2015;100(8):3140-8.

21. Cannon CP, Cariou B, Blom D, et al. Efficacy and safety of alirocumab in high cardiovascular risk patients with inadequately controlled hypercholesterolaemia on maximally tolerated doses of statins: the ODYSSEY COMBO II randomized controlled trial. Eur Heart J. 2015;36(19):1186-94.

22. Kereiakes DJ, Robinson JG, Cannon CP, et al. Efficacy and safety of the proprotein convertase subtilisin/kexin type 9 inhibitor alirocumab among high cardiovascular risk patients on maximally tolerated statin therapy: the ODYSSEY COMBO I study. Am Heart J. 2015;169(6):906-15.e13.

23. Farnier M, Jones P, Severance R, et al. Efficacy and safety of adding alirocumab to rosuvastatin versus adding ezetimibe or doubling the rosuvastatin dose in high cardiovascular-risk patients: the ODYSSEY OPTIONS II randomized trial. Atherosclerosis. 2016;244:138-46.

24. Ginsberg HN, Rader DJ, Raal FJ, et al. Efficacy and safety of alirocumab in patients with heterozygous familial hypercholesterolemia and LDL-C of $160 \mathrm{mg} / \mathrm{dl}$ or higher. Cardiovasc Drugs Ther. 2016;30(5):473-83.

25. Mager DE, Jusko WJ. General pharmacokinetic model for drugs exhibiting target-mediated drug disposition. J Pharmacokinet Pharmacodyn. 2001;28(6):507-32.

26. Djebli N, Martinez J-M, Lohan L, et al. Target-mediated drug disposition population pharmacokinetics model of alirocumab in healthy volunteers and patients: pooled analysis of randomized phase I/II/III studies. Clin Pharmacokinet. 2017;56(10):1155-71.

27. Martinez J-M, Brunet A, Hurbin F, et al. Population pharmacokinetic analysis of alirocumab in healthy volunteers or hypercholesterolemic subjects using a Michaelis-Menten approximation of a target-mediated drug disposition model-support for a Biologics License Application submission: Part I. Clin Pharmacokinet. 2018. https://doi.org/10.1007/s40262-018-0669-y.

28. Stein EA, Mellis S, Yancopoulos GD, et al. Effect of a monoclonal antibody to PCSK9 on LDL cholesterol. N Engl J Med. 2012;366(12):1108-18.

29. Teramoto T, Kobayashi M, Uno K, et al. Efficacy and safety of alirocumab in Japanese subjects (phase 1 and 2 studies). Am J Cardiol. 2016;118(1):56-63.

30. Rey J, Poitiers F, Paehler T, et al. Relationship between lowdensity lipoprotein cholesterol, free proprotein convertase subtilisin/kexin type 9, and alirocumab levels after different lipidlowering strategies. J Am Heart Assoc. 2016;5(6):e003323.

31. Roth EM, McKenney JM, Hanotin C, et al. Atorvastatin with or without an antibody to PCSK9 in primary hypercholesterolemia. N Engl J Med. 2012;367(20):1891-900.

32. McKenney JM, Koren MJ, Kereiakes DJ, et al. Safety and efficacy of a monoclonal antibody to proprotein convertase subtilisin/kexin type 9 serine protease, SAR236553/REGN727, in patients with primary hypercholesterolemia receiving ongoing stable atorvastatin therapy. J Am Coll Cardiol. 2012;59(25):2344-53.

33. Stein EA, Gipe D, Bergeron J, et al. Effect of a monoclonal antibody to PCSK9, REGN727/SAR236553, to reduce low-density lipoprotein cholesterol in patients with heterozygous familial hypercholesterolaemia on stable statin dose with or without ezetimibe therapy: a phase 2 randomised controlled trial. Lancet. 2012;380(9836):29-36.

34. Roth EM, Taskinen MR, Ginsberg HN, et al. Monotherapy with the PCSK9 inhibitor alirocumab versus ezetimibe in patients with hypercholesterolemia: results of a 24 week, double-blind, randomized phase 3 trial. Int J Cardiol. 2014;176(1):55-61.

35. Friedewald WT, Levy RI, Fredrickson DS. Estimation of the concentration of low-density lipoprotein cholesterol in plasma, without use of the preparative ultracentrifuge. Clin Chem. 1972;18(6):499-502.

36. Speth H. A Linux cluster for population pharmacokinetic analyses. Int J Clin Pharmacol Ther. 2004;42(3):189-90.

37. Jusko WJ, Ko HC. Physiologic indirect response models characterize diverse types of pharmacodynamic effects. Clin Pharmacol Ther. 1994;56(4):406-19.

38. Mayne J, Dewpura T, Raymond A, et al. Plasma PCSK9 levels are significantly modified by statins and fibrates in humans. Lipids Health Dis. 2008;11(7):22.

39. Guo YL, Zhang W, Li JJ. PCSK9 and lipid lowering drugs. Clin Chim Acta. 2014;1(437):66-71.

40. Dubuc G, Chamberland A, Wassef $\mathrm{H}$, et al. Statins upregulate PCSK9, the gene encoding the proprotein convertase neural apoptosis-regulated convertase-1 implicated in familial hypercholesterolemia. Arterioscler Thromb Vasc Biol. 2004;24(8):1454-9.

41. Raal F, Panz V, Immelman A, Pilcher G. Elevated PCSK9 levels in untreated patients with heterozygous or homozygous familial hypercholesterolemia and the response to high-dose statin therapy. J Am Heart Assoc. 2013;2(2):e000028.

42. Glerup S, Schulz R, Laufs U, Schluter KD. Physiological and therapeutic regulation of PCSK9 activity in cardiovascular disease. Basic Res Cardiol. 2017;112(3):32.

43. Turner BJ, Hollenbeak CS, Weiner M, Tang SS. A retrospective cohort study of the potency of lipid-lowering therapy and racegender differences in LDL cholesterol control. BMC Cardiovasc Disord. 2011;30(11):58.

44. Cholesterol Treatment Trialists Collaboration, Fulcher J, O'Connell R, et al. Efficacy and safety of LDL-lowering therapy among men and women: meta-analysis of individual data from 
174,000 participants in 27 randomised trials. Lancet. 2015;385(9976):1397-405.

45. Deedwania P, Stone PH, Bairey Merz CN, et al. Effects of intensive versus moderate lipid-lowering therapy on myocardial ischemia in older patients with coronary heart disease: results of the Study Assessing Goals in the Elderly (SAGE). Circulation. 2007;115(6):700-7.

46. Ghosh M, Galman C, Rudling M, Angelin B. Influence of physiological changes in endogenous estrogen on circulating PCSK9 and LDL cholesterol. J Lipid Res. 2015;56(2):463-9.

47. Abrams R, Phair R, McQuade S, et al. Discovery of novel cholesterol metabolism biology to explain gender difference of PCKS9 inhibitors through quantitative systems pharmacology modeling. In: Poster presented at the American Association of pharmaceutical scientists annual meeting, 12-15 November, 2017; San Diego (CA). 2017. http://abstracts.aaps.org/Verify/ AAPS2017/PosterSubmissions/M6073.pdf. Accessed 19 April 2018.

48. Gibbs JP, Doshi S, Kuchimanchi M, et al. Impact of target-mediated elimination on the dose and regimen of evolocumab, a human monoclonal antibody against proprotein convertase subtilisin/kexin type 9 (PCSK9). J Clin Pharmacol. 2017;57(5):616-26.

49. Kim J, Ahn BJ, Chae HS, et al. A population pharmacokineticpharmacodynamic model for simvastatin that predicts low-density lipoprotein-cholesterol reduction in patients with primary hyperlipidaemia. Basic Clin Pharmacol Toxicol. 2011;109(3):156-63. 